\title{
The interaction factor and directionality in wave energy arrays
}

\author{
H.A. Wolgamot ${ }^{a, *}$, P.H. Taylor ${ }^{a}$, R. Eatock Taylor ${ }^{a}$ \\ ${ }^{a}$ Department of Engineering Science, University of Oxford, Parks Road, Oxford, OX1 3PJ, \\ United Kingdom
}

\begin{abstract}
This paper considers arrays of wave energy devices consisting of oscillating rigid bodies undergoing optimum displacements in regular waves. A previously published identity concerning the directional behaviour of the interaction factor, $q$, (which characterises an important effect of the array configuration in the context of point absorber theory) is shown to be valid under more general conditions. In fact, this identity is shown to be a consequence of a more fundamental result. Boundary element hydrodynamic calculations carried out on simple 3-member arrays are shown to be consistent with these theoretical proofs. Some aspects of the behaviour of the arrays are also investigated.

Keywords:

wave energy, array, interaction factor
\end{abstract}

\section{Introduction}

As wave energy moves to commercial scale, arrays of wave energy devices may be expected to become common. Devices in an array experience forces due to waves scattered and radiated from other devices, with commensurate impacts

\footnotetext{
${ }^{*}$ Corresponding author. Tel.: +44 758055 8106, Fax: +44 1865273010

Email addresses: hugh.wolgamot@eng.ox.ac.uk (H.A. Wolgamot), paul.taylor@eng.ox.ac.uk (P.H. Taylor), r.eatocktaylor@eng.ox.ac.uk (R. Eatock Taylor)
} 
on the power that may be absorbed. A special case of the array situation arises for devices consisting of a number of floats connected to a single structure, a number of which are currently under development - in this case the device spacings are likely to be smaller than in the general case.

Studies of wave energy arrays have been of interest since the early days of wave energy research. Budal (1977) commenced in this direction and introduced two concepts which have been much used in subsequent work. The first was the treatment of wave energy devices as 'point absorbers' - devices small enough for the scattered wave to be neglected and only the radiated wave retained in the analysis. The second concept was the interaction factor, which may be defined as the ratio of the maximum power which may be absorbed by an array of identical wave energy devices to the maximum power available from the same number of individual members of the array acting independently of their neighbours.

Subsequently Evans (1980) and Falnes (1980) independently derived some fundamental results for array problems; firstly, a general theory for power absorption by arrays of oscillating bodies in regular waves and second, derived from the general theory, an expression for maximum power absorption by arrays. Both works then went on to analyse arrays using the point absorber assumption and were able to find interaction factors in excess of 1 .

To achieve maximum power absorption according to the theory of Evans and Falnes, an optimum motion condition must be satisfied and it was swiftly recognised that optimum device oscillation amplitudes could become so large as to be inconsistent with linear theory. Thomas and Evans (1981) therefore examined linear arrays of 5 hemispheres using the point absorber approximation, and studied cases where oscillation amplitudes were limited to twice or three times the incident wave amplitude. In cases where these limits prevented optimum motions from being achieved, it was reported that power absorption 
could be significantly reduced.

The impact of wave direction on power absorption by an array was considered by McIver (1994), who found that the interaction factor could vary significantly with incoming wave direction. Fitzgerald and Thomas (2007) proceeded further in this direction, analysing arrays of five hemispheres using point absorber theory and studying variation of both array geometry and incoming wave direction. In the course of this study it was proved (Fitzgerald (2006)) that under point absorber theory the average of the interaction factor over all incident wave directions 0 to $2 \pi$ is equal to unity, and this was confirmed by numerical results. This result was significant because it showed that arrays with high values of the interaction factor at some wave heading must be associated with unfavourable values at some other wave heading.

Child and Venugopal (2010) optimised the layout of arrays of five truncated cylinders using a semi-analytical approach which included scattering from the devices. All devices were assigned the same power-take off (damping and stiffness values), meaning that the motions were again non-optimal. In the course of this analysis Child and Venugopal (2010) considered the identity presented by Fitzgerald and Thomas (2007) (with a modified non-optimal interaction factor) and found that it did not hold exactly, attributing this mainly to the nonoptimality mentioned above, but also to the fact that point-absorber theory was not employed.

The usefulness of the identity of Fitzgerald and Thomas (2007) is apparently limited by the bounds of point absorber theory - in a study comparing different methods of analysis for wave energy arrays Mavrakos and McIver (1997) suggested that the point absorber approximation would calculate accurate interaction factors only for non-dimensionalised wavenumbers, $k a$, less than 0.8 . Therefore, in this paper we revisit this identity for the interaction factor and 
show that it in fact holds outside point absorber theory; indeed, we show that this identity is itself the consequence of what appears to be a more fundamental and widely applicable result. In later sections we also provide numerical results which support this proof and illustrate various influences of constructive and destructive interference on optimum power in the general case of an array of scattering devices.

\section{Theory}

\subsection{The Interaction Factor}

The interaction factor concept has been much used in studies of wave energy arrays. We restate the definition of the interaction factor, $q$

$$
q(\beta)=\frac{P_{N, \max }}{N P_{1, \max }}
$$

where $P_{N, \max }$ is the maximum power absorbed by an array of $N$ identical devices, $P_{1, \max }$ the power absorbed by a single such device in isolation and $\beta$ is the incident wave direction. It is important to emphasise that in this paper we only consider $q$ defined as a ratio of these optimum values - many similar metrics have been deployed in wave energy studies where non-optimum power is the subject of study.

Using point absorber theory to analyse arrays of heaving hemispheres, Fitzgerald and Thomas (2007) proved that

$$
\frac{1}{2 \pi} \int_{0}^{2 \pi} q(\beta) \mathrm{d} \beta=1
$$

This result motivated the present work seeking to extend it outside the zone of applicability of point absorber theory. 


\subsection{Wave Energy Theory}

This paper treats only regular waves and adopts linear wave theory throughout - the flow is assumed to be inviscid, incompressible and irrotational, and the waves and body motions are of small amplitude. An important quantity in any wave power study is the incoming wave power transport

$$
J=\frac{\rho g^{2} D(k h)}{4 \omega} A^{2}
$$

which is the power per unit width of wave front transported by a wave of angular frequency $\omega$, wavenumber $k$ and amplitude $A$ (Falnes (2002)), where $\rho$ is the density of water, $g$ the acceleration due to gravity and

$$
D(k h)=\left\{1+\frac{2 k h}{\sinh 2 k h}\right\} \tanh k h
$$

is a function of the depth $h$ which is asymptotic to 1 in deep water.

We now consider an array of independently oscillating rigid bodies absorbing wave energy through $N$ oscillating modes, where $N$ may be equal to or greater than the number of devices, so that each device may operate in more than one mode (up to the physical limit of 6 ). The time-averaged power that can be absorbed by a single oscillator (i.e. a single mode of one device) is equal to (Falnes (2002))

$$
P_{i}=\frac{1}{4}\left(F_{e, i} u_{i}^{*}+F_{e, i}^{*} u_{i}\right)-\frac{1}{4} \sum_{j=1}^{N}\left(Z_{i j} u_{j} u_{i}^{*}+Z_{i j}^{*} u_{j}^{*} u_{i}\right)
$$

where $F_{e, i}$ is the complex amplitude of the exciting force on the oscillator, $u_{i}$ the complex amplitude of the velocity of the oscillator, ${ }^{*}$ denotes complex conjugate and $Z_{i j}$ is the radiation impedance (with real part equal to the radiation damping and imaginary part proportional to the added mass). The power absorbed 
by the whole array may be expressed as

$$
P_{N}=\frac{1}{4}\left(\boldsymbol{u}^{\dagger} \boldsymbol{F}_{\boldsymbol{e}}+\boldsymbol{F}_{e}^{\dagger} \boldsymbol{u}\right)-\frac{1}{2} \boldsymbol{u}^{\dagger} \boldsymbol{B} \boldsymbol{u}
$$

where $\boldsymbol{F}_{\boldsymbol{e}}, \boldsymbol{u}$ are respectively the $N \times 1$ vectors of complex amplitudes of the exciting forces and body velocities, $\boldsymbol{B}$ is the $N \times N$ radiation damping matrix and $^{\dagger}$ denotes complex conjugate transpose. From (6) the maximum power that may be absorbed by the array, originally presented by Evans (1980) and Falnes (1980), may be derived as

$$
P_{N, \max }=\frac{1}{8} \boldsymbol{F}_{e}^{\dagger} \boldsymbol{B}^{-\mathbf{1}} \boldsymbol{F}_{\boldsymbol{e}}
$$

which occurs when the optimum condition

$$
\boldsymbol{u}_{\text {opt }}=\frac{1}{2} \boldsymbol{B}^{-1} \boldsymbol{F}_{\boldsymbol{e}}
$$

is satisfied (provided the inverse $\boldsymbol{B}^{-\mathbf{1}}$ exists).

It is possible for the damping matrix to be singular; this is the case, for example, for an axisymmetric body moving in surge and pitch, because the two modes radiate the same wave pattern (Newman (1976)). In fact, for an axisymmetric body, the damping matrix is singular if more than three modes are involved, as there are only three unique angular variations possible in the outgoing circular wave (Falnes (2002)). It is also possible for the damping matrix to become singular at certain frequencies for some body geometries, as identified, for example, by Thomas and Evans (1983) - in such cases the body may oscillate with no radiation to infinity. Finally, the damping matrix for multiple degreeof-freedom systems will tend to become singular in the low-frequency limit (e.g. Newman (1983)). Nevertheless, in most cases of interest it is expected that the 
damping matrix will be positive definite - this assumption has been made in a great majority of such wave power studies and will similarly be employed here.

A useful way of thinking about wave energy is to consider power absorption as destructive interference between waves radiated from a device and the incident and scattered waves present when the device is not moving (as these may be separated under linear theory). The optimum in (7) must therefore correspond to optimum wave cancellation. It is apparent that the singular behaviour of $\boldsymbol{B}$ in the case of a device moving in two modes with the same radiated wave pattern is unlikely to be of concern - the device can absorb no more power with two modes than with one, as the interference pattern will be the same, so the second mode is not useful. The interference approach will be useful in later discussion.

\subsection{Single axisymmetric devices}

Single axisymmetric devices are the simplest (3-dimensional) bodies to analyse, and so classical results for power absorption have been known for some time. For example, the maximum power that may be absorbed by a single heaving axisymmetric device may be shown (using (7) or otherwise) to be (e.g. Evans $(1980))$

$$
P_{1, \max }=J / k
$$

which occurs when the body is at resonance; this is independent of the direction of the incoming wave.

Similarly, the result for a surging and swaying device is

$$
P_{1, \max }=2 J / k
$$


while for a device which is surging only the result is

$$
P_{1, \max }=2 J \cos ^{2} \beta / k
$$

where surge and sway are along the $x-$ and $y$-axes and $\beta$ is measured from these axes in the conventional manner. Note that the surging and swaying axisymmetric device will effectively be surging into the wavefront for all wave approach directions; in this paper, however, we define surge using the fixed coordinate system rather than the incident wave direction.

\subsection{Directional considerations}

Consider the definition of $q$, given in equation (1). It is apparent that for a single heaving axisymmetric body the power absorbed is not a function of incident wave angle (confirmed by (9)). Therefore, any interest in the directional behaviour of $q$ for an array of such devices must reside in the array power term in the numerator. We therefore consider the integral

$$
H=\frac{1}{2 \pi} \int_{0}^{2 \pi} \frac{1}{8} \boldsymbol{F}_{\boldsymbol{e}}^{\dagger}(\beta) \boldsymbol{B}^{-1} \boldsymbol{F}_{\boldsymbol{e}}(\beta) \mathrm{d} \beta
$$

in which it is apparent that the main difficulty resides in the inverse.

Using the fact that $\boldsymbol{B}$ is symmetric and (by assumption, see Section 2.2) positive definite, we may adopt the Cholesky decomposition and write $\boldsymbol{B}$ as the product of an upper triangular matrix and its transpose

$$
\boldsymbol{B}=\boldsymbol{U}^{T} \boldsymbol{U}
$$

so

$$
B^{-1}=U^{-1} U^{T^{-1}}
$$


If we define

$$
\boldsymbol{Q}(\beta)=\boldsymbol{U}^{\boldsymbol{T}^{-1}} \boldsymbol{F}_{\boldsymbol{e}}(\beta)
$$

then

$$
H=\frac{1}{16 \pi} \int_{0}^{2 \pi} \boldsymbol{Q}^{\dagger}(\beta) \boldsymbol{Q}(\beta) \mathrm{d} \beta
$$

noting that $\boldsymbol{B}$, and therefore $\boldsymbol{U}$, is real.

We now introduce an important, and well known, reciprocity relation. As shown by Newman (1976) and others, it is possible to express the radiation damping coefficients in terms of the exciting forces in waves at an angle of incidence $\beta$

$$
\boldsymbol{B}=\frac{k}{16 \pi J} \int_{0}^{2 \pi} \boldsymbol{F}_{\boldsymbol{e}}(\beta) \boldsymbol{F}_{\boldsymbol{e}}^{\dagger}(\beta) \mathrm{d} \beta .
$$

We may then use this reciprocity relation to write

$$
\boldsymbol{U}^{\boldsymbol{T}^{-1}} \boldsymbol{B} \boldsymbol{U}^{-\mathbf{1}}=\frac{k}{16 \pi J} \int_{0}^{2 \pi} \boldsymbol{Q}(\beta) \boldsymbol{Q}^{\dagger}(\beta) \mathrm{d} \beta=\boldsymbol{I}
$$

(where $\boldsymbol{I}$ is the identity matrix) which shows that

$$
\frac{k}{16 \pi J} \int_{0}^{2 \pi} Q_{i}(\beta) Q_{j}^{*}(\beta) \mathrm{d} \beta=\left\{\begin{array}{l}
1, i=j \\
0, i \neq j
\end{array}\right.
$$

where $Q_{i}(\beta)$ are the elements of vector $\boldsymbol{Q}(\beta)$. Now, (16) shows that

$$
H=\frac{1}{16 \pi} \int_{0}^{2 \pi} \sum_{i=1}^{N} Q_{i}^{*}(\beta) Q_{i}(\beta) \mathrm{d} \beta
$$

and therefore

$$
H=\frac{1}{2 \pi} \int_{0}^{2 \pi} P_{N, \max }(\beta) \mathrm{d} \beta=N \frac{J}{k} .
$$

This result has been derived without any assumption of body size or shape. 


\subsection{Consequences}

It is trivial that, for a single axisymmetric device, equation (21) is in agreement with equations (9), (10) and (11). More significantly, it is also apparent that, for arrays of heaving axisymmetric devices the result

$$
\frac{1}{2 \pi} \int_{0}^{2 \pi} q(\beta) \mathrm{d} \beta=1
$$

must hold regardless of body dimension. This will also hold for arrays of axisymmetric devices moving in surge and sway, or indeed, for any combination of three or fewer modes for which the power absorbed by an isolated device is invariant with direction.

For a single non-axisymmetric device equation (21) indicates that the maximum power that the device can absorb, averaged over all directions, is the same as for an axisymmetric device. This is perhaps not a surprising result, since if the device can radiate more wave energy than an axisymmetric body in one direction, better cancellation of the incident and diffracted wave may occur,

however, in some other direction there will be less radiated energy than from the axisymmetric body and accordingly less favourable interference. From the derivation it is apparent that (21) applies to arrays of arbitrary oscillating body devices and is more fundamental than (22) since it can be easily applied to heterogenous arrays of non-axisymmetric devices. In these cases, it is not clear that the interaction factor concept can be so easily extended, as the performance of each isolated device may vary with incoming wave direction.

\section{Hydrodynamic Calculations}

\subsection{DIFFRACT}

To provide numerical evidence that the result above applies to optimised arrays, some hydrodynamic calculations on simple arrays have been performed. 
In order to include all the relevant linear hydrodynamics, the boundary element hydrodynamics program DIFFRACT was used for this task. DIFFRACT originated in the work of Chau (1989) and has been extended and extensively validated over the ensuing years (e.g. Zang et al. (2006)). This program can solve the diffraction and radiation problems for fixed and moving bodies at first and second-order (though only linear calculations are performed here). For linear calculations the submerged surfaces and inner water planes of the bodies must be meshed with quadratic elements (an example mesh for one array studied here is shown in Figure 14). Boundary element programs are now a common tool for analysing arrays of wave energy devices; studies that have employed this technique include, for example, Thomas et al. (2008).

Following a trend of increasing complexity, the following sections present results for arrays of three devices; first hemispheres (as simple axisymmetric devices) heaving and then moving more generally, and then arbitrary devices. In between, the opportunity is taken to expand some results from the arrays of hemispheres which are of interest.

\subsection{Heaving hemispheres}

Four different configurations of hemispheres of radius $a$ were analysed; three triangular layouts and a linear case, as shown in Figure 1. In all cases the water depth was $10 a$.

\subsubsection{Convergence}

The first array analysed was Configuration A - an array of hemispheres laid out with centres at the vertices of an equilateral triangle. Figure 2 shows the variation of the interaction factor with incident wave direction for three different non-dimensional wave numbers $k a$ and it may be seen that the interaction factor oscillates about unity. Taking advantage of symmetry, the interaction factor is 
shown only for the range $0^{\circ}$ to $60^{\circ}$ - the directional resolution of the calculations is $3.75^{\circ}$.

To confirm that these results are not merely reproducing a point absorber analysis, point absorber results generated using the same method as Fitzgerald and Thomas (2007) are also displayed. The results are consistent with the point absorber limit of $k a=0.8$ suggested by Mavrakos and McIver (1997) as cited in Section 1.

As with any BEM analysis, it is important to establish mesh convergence. Hence the equilateral array was analysed using three meshes, with 32, 168 and 306 elements per hemisphere respectively. Convergence of the interaction factor for two wave directions at the same three incident wavenumbers is displayed in Table 1.

\begin{tabular}{|c|c|c|c|}
\hline$k a$ & Mesh & $q\left(0^{\circ}\right)$ & $q\left(30^{\circ}\right)$ \\
\hline \multirow{3}{*}{0.5} & 32 & 0.9829 & 0.9987 \\
& 168 & 0.9847 & 1.0008 \\
& 306 & 0.9848 & 1.0008 \\
\hline \multirow{3}{*}{1.0} & 32 & 1.1975 & 0.7136 \\
& 168 & 1.1989 & 0.7147 \\
& 306 & 1.1989 & 0.7147 \\
\hline \multirow{3}{*}{1.5} & 32 & 0.8110 & 1.2057 \\
& 168 & 0.8111 & 1.2092 \\
& 306 & 0.8111 & 1.2095 \\
\hline
\end{tabular}

Table 1: Convergence of $q$ (heave-only) at discrete wavenumbers (Configuration A). The 'Mesh' column refers to the number of quadrilateral elements per hemisphere.

Of more interest is numerical convergence of the integrated quantity (22) towards unity. Numerical integration over the angular range was carried out using a simple Simpson's Rule approach. Convergence of this quantity for the same three meshes is shown in Table 2 and satisfactory behaviour may be observed. 


\subsubsection{Various Configurations}

The variation of the interaction factor with incoming wave direction for the Configurations $\mathrm{B}$ and $\mathrm{C}$ are shown in Figures 3 and 4, respectively. Having studied convergence for Configuration A, the finest mesh density was adopted here. Again, the oscillatory behaviour of the interaction factor about unity is observed. Possessing less symmetry, the results for these arrays must be shown over a wider directional range $-180^{\circ}$ and $360^{\circ}$, respectively. The directional resolution of the calculations was $5^{\circ}$ in each case. Under the point absorber approximation Fitzgerald (2006) proved that $q(\beta)=q(\beta+\pi)$ - it may be seen that while the plots broadly mimic this feature, it clearly does not hold exactly when scattering is included.

Values of the identity (22) calculated for the three triangular array cases displayed above are presented in Table 3. Also presented here are the equivalent results for a linear array of such hemispherical devices, with centre-to-centre spacing $4 a$. It may be seen that the desired value of unity is reproduced in all cases with considerable accuracy. The largest errors $(0.06 \%)$ are for the highest frequency, which would be expected - the shorter waves are less well resolved for the same mesh size.

\begin{tabular}{|c|c|c|}
\hline$k a$ & Mesh & $\frac{1}{2 \pi} \int_{0}^{2 \pi} q(\beta) \mathrm{d} \beta$ \\
\hline \multirow{3}{*}{0.5} & 32 & 0.9979 \\
& 168 & 1.0001 \\
& 306 & 1.0001 \\
\hline \multirow{3}{*}{1.0} & 32 & 0.9998 \\
& 168 & 0.9999 \\
& 306 & 1.0000 \\
\hline \multirow{3}{*}{1.5} & 32 & 0.9944 \\
& 168 & 0.9991 \\
& 306 & 0.9994 \\
\hline
\end{tabular}

Table 2: Convergence of quantity (22) (heave-only) for Configuration A. 


\begin{tabular}{|c|c|c|}
\hline Config. & $k a$ & $\frac{1}{2 \pi} \int_{0}^{2 \pi} q(\beta) \mathrm{d} \beta$ \\
\hline & 0.5 & 1.0001 \\
$\mathrm{~A}$ & 1.0 & 1.0000 \\
& 1.5 & 0.9994 \\
\hline & 0.5 & 1.0001 \\
$\mathrm{~B}$ & 1.0 & 0.9999 \\
& 1.5 & 0.9995 \\
\hline & 0.5 & 1.0001 \\
$\mathrm{C}$ & 1.0 & 0.9999 \\
& 1.5 & 0.9994 \\
\hline & 0.5 & 1.0001 \\
$\mathrm{D}$ & 1.0 & 0.9999 \\
& 1.5 & 0.9994 \\
\hline
\end{tabular}

Table 3: Numerical values of quantity (22) obtained from DIFFRACT analyses of the four heave-only systems.

\subsubsection{Array behaviour}

Having considered the 'global' behaviour of the array in terms of the interaction factor it is now useful to consider some of the 'internal' properties of the array. For this purpose we return to Configuration A, the equilateral triangle. It is well known that the optimum amplitudes given by equation (8) can be so large as to violate the assumptions of linear theory and the practicalities of a real device. The heave excursions of the three devices are plotted in Figure 5, non-dimensionalised by the wave amplitude $A$. It is well known that larger devices need to move less than smaller devices to absorb optimal power - this may be clearly seen here. For the wavenumbers $k a=1.0$ and 1.5, where the devices are relatively large, the maximum heave remains less than about twice the wave amplitude, suggesting that for these conditions the assumption of linear dynamics is not unreasonable. Figure 6 displays the phases of the heave displacements for $k a=1.0$. It is evident from this plot, and from Figure 5, that the motions of the devices are unique, except where symmetry applies.

Also of interest is the partition of power amongst oscillators, which may 
be calculated simply using equation (5) provided that the added mass matrix is known (knowledge of the power take-off mechanism is not required). The added mass matrix is a standard output from DIFFRACT, and the calculation is therefore straightforward. Figure 7 shows the fraction of total power absorbed by each hemisphere in Configuration A, for the same 3 wavenumbers and over the same range of wave directions, $0^{\circ}$ to $60^{\circ}$. The expected symmetries may be observed. It is of interest that the devices do not, in general, absorb equal amounts of power. For the case $k a=1.0$ the power value for one device becomes negative (but small) as the incident wave angle approaches $60^{\circ}$. This of course indicates that the device is supplying power into the wavefield rather than absorbing it. Obviously such a situation would not be desirable in a real device, but merely results from the hydrodynamic optimality condition applied here.

DIFFRACT may also be used to investigate the behaviour of the free surface around these arrays. Figure 8 shows the amplitude of the wave field around the devices of Configuration $\mathrm{C}$ when the devices are fixed and regular waves with a $k a$ value of unity are incident from $0^{\circ}$ (the direction vector is defined counterclockwise from the $x$-axis as normal, such that here the waves propagate from left to right). As the devices are fixed, only the incident and scattered waves are shown. It is apparent from this plot that scattering is significant, and that, as expected, the point absorber approximation is not appropriate. The following plot, Figure 9, shows the wave field around the devices when they are heaving with optimum motions as given by equation (8). This requires inserting external stiffness and damping values into DIFFRACT for each device, such that the desired motions are achieved in response to the incident wave (negative stiffness values were used). A quick comparison of the two figures will reveal that the 'shadow' zone of low wave amplitude downwave of the devices in Figure 8 has been enhanced in Figure 9. This must be due to cancellation of the waves 
passing the devices by the radiated wave, and indicates that power has been extracted by the devices. Figures 8 and 9 both show beaming and interference patterns in what appear to be 5 major directions.

Having discussed the influence of the radiated wave it is of interest to examine it - the radiated wave was therefore isolated by subtracting the (complexvalued) wave fields from the two previous cases, yielding the amplitudes presented here in Figure 10. This is, of course, the free surface that would be seen were the devices oscillated with optimum motions (for $0^{\circ}$ ) on otherwise quiescent water. It is evident that there is a strong beam of wave energy being radiated downwave of the devices, consistent with the previous argument about interference. Further, there are 5 other radiated beams apparent in this Figure, matching the beaming observed in the cases above with incident waves. However, in this case, there is no intereference pattern present in the beams.

Waves incident on Configuration $\mathrm{C}$ from $0^{\circ}$ at $k a=1.0$ correspond to a peak in the interaction factor, as may be seen in Figure 4. It is evident from this same plot that waves incident from $180^{\circ}$ similarly correspond to a favourable interaction factor, and waves from $135^{\circ}$ correspond to a low value of the interaction factor. In the same manner as above, the optimum radiated wave for incident waves from $180^{\circ}$ is shown in Figure 11 and it is apparent that a very similar structure exists - a strong beam is radiated downwave. Conversely, in Figure 12, which displays the radiated wave for incident waves from $135^{\circ}$, it is apparent that the radiated wave in the downwave direction is very much weaker. It seems that this layout of devices is not able, even with optimum body amplitudes and phases, to create a radiated wave that interferes well with the incident and scattered waves. These observations are in accord with those of Folley and Whittaker (2009) who considered radiation patterns from point absorber arrays. 


\subsection{Surging and swaying hemispheres}

In Section 2.5 we stated that equation (22) should apply to axisymmetric devices moving in modes other than heave. One combination of power-absorbing modes satisfying the criteria set down there is surge and sway. Hence Figure 13 displays the variation of the interaction factor with incident wave direction for surging and swaying hemispheres laid out in Configuration A. Table 4 displays the values thus calculated from equation (22). The results are again consistent with the proof, but it is of interest to note that in this case the largest deviation from unity occurs for the lowest wavenumber - this is thought to be because the damping matrix is moving closer to the range in which singular behaviour is observed, as discussed in Section 2.2.

\begin{tabular}{|c|c|}
\hline$k a$ & $\frac{1}{2 \pi} \int_{0}^{2 \pi} q(\beta) \mathrm{d} \beta$ \\
\hline 0.5 & 1.0008 \\
\hline 1.0 & 1.0001 \\
\hline 1.5 & 1.0004 \\
\hline
\end{tabular}

Table 4: Numerical values of identity (22) obtained from DIFFRACT analysis of Configuration A in surge and sway.

\subsection{Arbitrary bodies}

To support the claim that the result (21) is valid for general device shapes and modes DIFFRACT was used to investigate power absorption from a heterogeneous array. In parallel with the calculations above, a three-device array was chosen. The three device shapes selected were a hemisphere, an ellipsoid and an elliptical cylinder positioned as shown in Figure 14 and Figure 15. Figure 14 is oriented to show the body shapes and DIFFRACT mesh clearly.

The ellipsoid, hemisphere and elliptical cylinder were then allowed to absorb power in one mode each; heave, surge and sway respectively. Optimal power absorption normalised by $N J / k$ (where $N$ is 3 ) is shown in Figure 16, and it 
is clear that there is very strong directional variation in this case. Even for the longest waves $(k a=0.5)$ the point absorber property $q(\beta)=q(\beta+\pi)$ is not satisfied for this array. The integrated values analogous to the interaction factor are displayed in Table 5 and again the results reproduce the expected values well. Such calculations have been performed for this array with rotational modes included and the results found to be similarly consistent with the theory.

\begin{tabular}{|c|c|}
\hline$k a$ & $\frac{k}{N J} \frac{1}{2 \pi} \int_{0}^{2 \pi} P_{N, \max }(\beta) \mathrm{d} \beta$ \\
\hline 0.5 & 1.0004 \\
\hline 1.0 & 1.0000 \\
\hline 1.5 & 1.0001 \\
\hline
\end{tabular}

Table 5: Calculated values of (21) for a heterogeneous array.

\section{Conclusions}

The identity (22) has been shown to be valid for axisymmetric devices moving in heave, or other combinations of modes compatible with the definition of $q$, regardless of wavenumber/body size. Further, a more fundamental result, equation (21), has been shown to underlie this identity, and to be generally applicable to arrays of oscillating rigid bodies. These identities are important as they indicate that for any wavenumber a peak in the power absorption curve at some incident wave angle must be associated with less absorption at other wave headings. This is significant for array design - matching the width of a peak in the $q$ curve to the range of expected incident wave directions would be a valuable consideration for a new array.

This paper has considered only optimum motions and regular waves. Although in this paper many examples involve motions consistent with the theoretical framework, it is clear that in some cases optimum motions will not be consistent with linear theory for examples of practical energy extraction. Child 
and Venugopal (2010) found that the identity (22) was approximated quite well for some arrays with motions not fully optimised in regular waves. The results shown here rely on the optimum in equation (7) - achieving this for each frequency component in an irregular sea is of theoretical interest and worthy of further study, but is unlikely to occur in real devices. Hence it will also be of interest to investigate how closely equations (21) and (22) are reproduced in sub-optimal arrays in irregular waves. Despite the limitations listed, it is thought that knowledge of the behaviour of optimum power absorption with varying incident regular wave direction is a valuable tool.

\section{Acknowledgements}

An early version of this work was presented at the 2011 International Workshop on Water Waves and Floating Bodies in Athens. We are very grateful to Professor David Evans who during the Workshop discussions suggested an extension of our initial result, leading to the general identity (21). We are also grateful for discussions on this topic with Dr Colm Fitzgerald. H. Wolgamot was supported by a University of Oxford Clarendon Fund Scholarship.

Budal, K., 1977. Theory for absorption of wave power by a system of interacting bodies. J. Ship Res. 21, 248-253.

Chau, F.P., 1989. The second order velocity potential for diffraction of waves by fixed offshore structures. Ph.D. thesis. University College London.

Child, B.F.M., Venugopal, V., 2010. Optimal configurations of wave energy device arrays. Ocean Eng. 37, 1402-1417.

Evans, D.V., 1980. Some analytic results for two- and three-dimensional waveenergy absorbers, in: Count, B. (Ed.), Power from Sea Waves. Academic Press, pp. 213-249. 
Falnes, J., 1980. Radiation impedance matrix and optimum power absorption for interacting oscillators in surface waves. Appl. Ocean Res. 2, 75-80.

Falnes, J., 2002. Ocean waves and oscillating systems. Cambridge University Press.

Fitzgerald, C., 2006. Optimal Configurations of Arrays of Wave Power Devices. Master's thesis. National University of Ireland, Cork.

Fitzgerald, C., Thomas, G., 2007. A preliminary study on the optimal formation of an array of wave power devices, in: Proceedings of the 7th European Wave and Tidal Energy Conference.

Folley, M., Whittaker, T.J.T., 2009. The effect of sub-optimal control and the spectral wave climate on the performance of wave energy converter arrays. Appl. Ocean Res. 31, 260-266.

Mavrakos, S.A., McIver, P., 1997. Comparison of methods for computing hydrodynamic characteristics of arrays of wave power devices. Appl. Ocean Res. $16,61-69$.

McIver, P., 1994. Some hydrodynamic aspects of arrays of wave energy devices. Appl. Ocean Res. 19, 283-291.

Newman, J.N., 1976. The interaction of stationary vessels with regular waves, in: Proceedings of the 11th Symposium on Naval Hydrodynamics, pp. 491501.

Newman, J.N., 1983. The low-frequency limit for wave-power systems. Appl. Ocean Res. 5, 38-44.

Thomas, G.P., Evans, D.V., 1981. Arrays of three dimensional wave-energy absorbers. J. Fluid Mech. 108, 67-88. 
Thomas, G.P., Evans, D.V., 1983. A hydrodynamic model of a submerged lenticular wave energy device. Appl. Ocean Res. 5, 69-79.

Thomas, S., Weller, S., Stallard, T., 2008. Float response within an array: numerical and experimental comparison, in: 2nd International Conference on Ocean Energy (ICOE2008).

Zang, J., Gibson, R., Taylor, P.H., Eatock Taylor, R., Swan, C., 2006. Second order wave diffraction around a fixed ship-shaped body in unidirectional steep waves. J. Offshore Mech. and Arctic Eng. 128, 89-99.
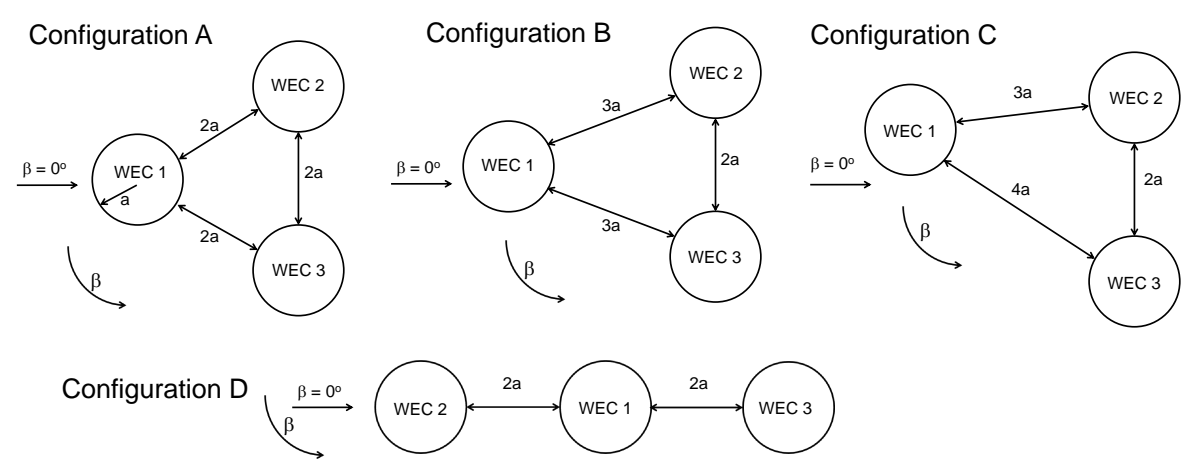

Figure 1: Plan-view layouts of 3-device array configurations. The devices are labelled 'WEC' for 'Wave Energy Converter'. 


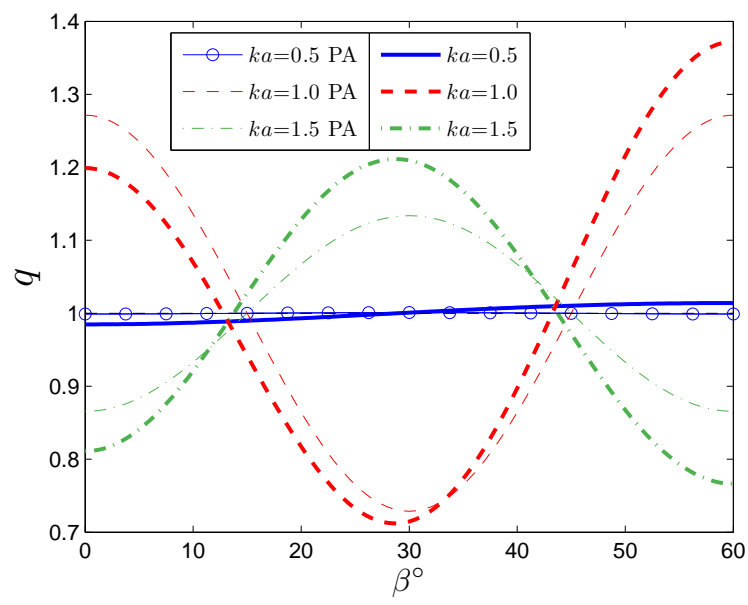

Figure 2: Heave-only $q$ factor for Configuration A (including point absorber results labelled PA).

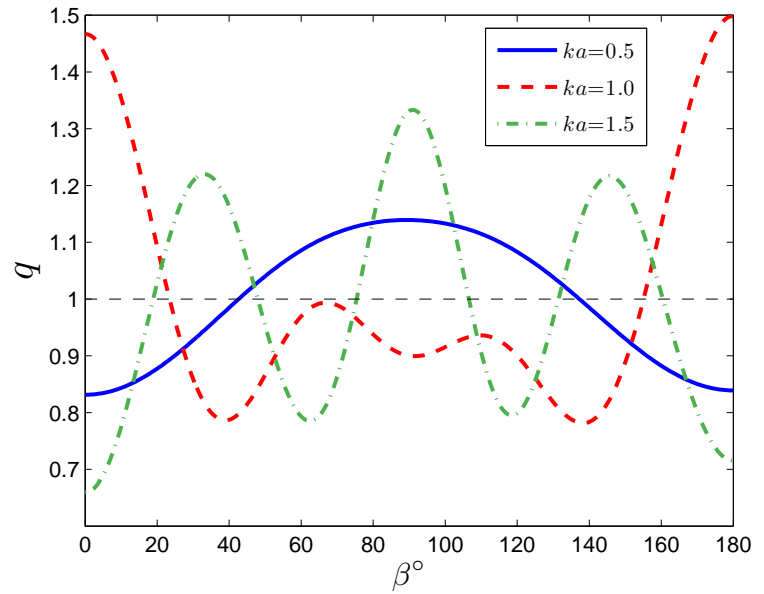

Figure 3: Heave-only $q$ factor for Configuration B (isosceles triangle). 


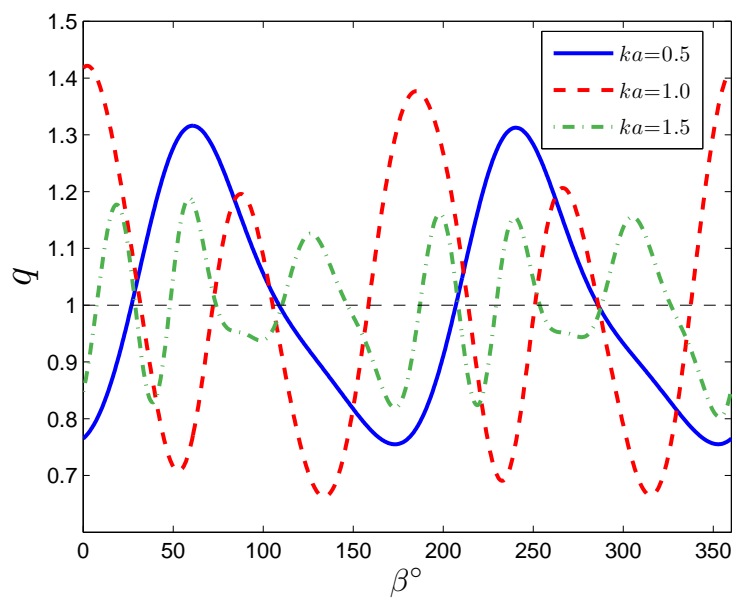

Figure 4: Heave-only $q$ factor for Configuration C (scalene triangle).

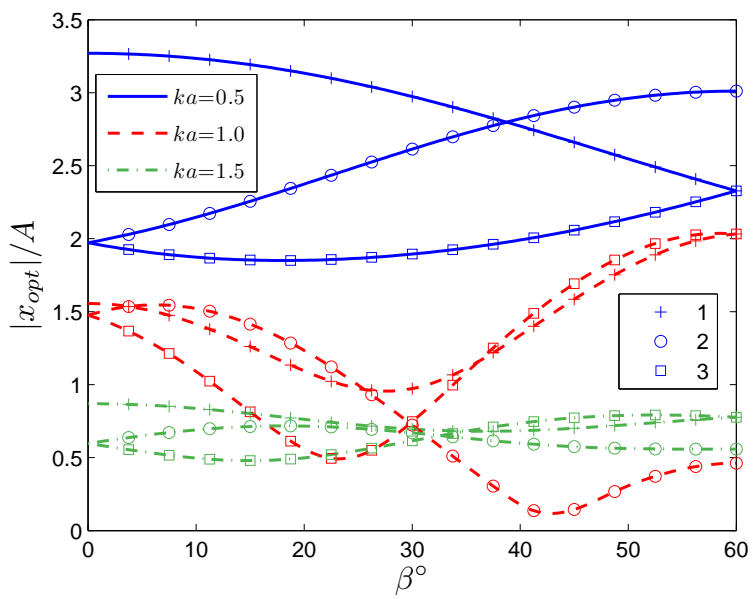

Figure 5: Amplitude of heave displacement of devices in Configuration A number labels identify the individual devices. 


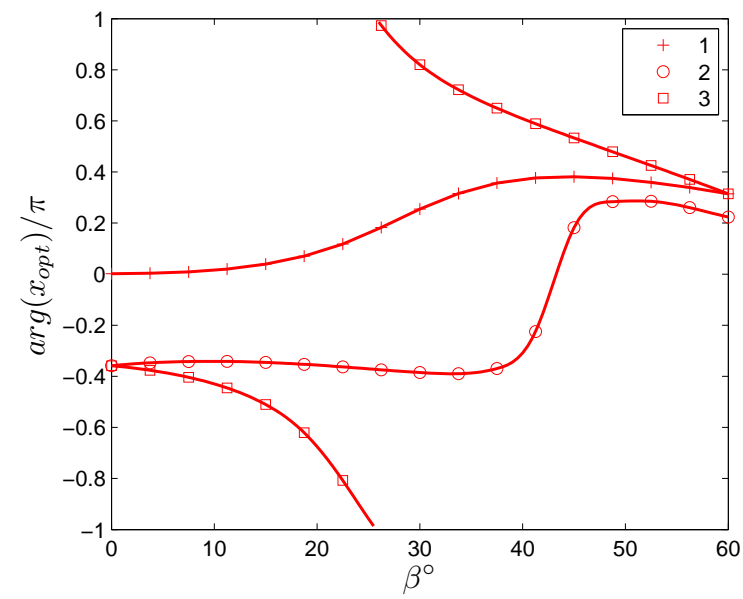

Figure 6: Phase of heave displacements for Configuration A (equilateral triangle), $k a=1.0$.

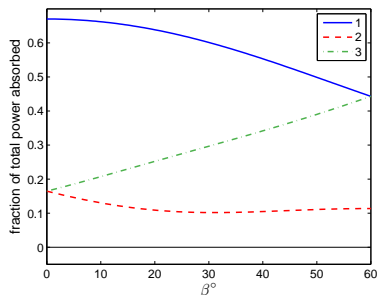

(a) $k a=0.5$

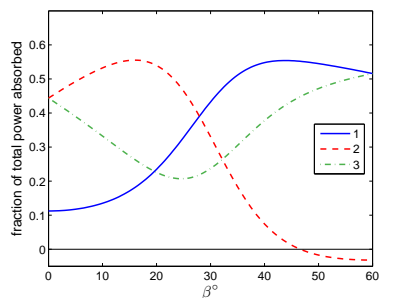

(b) $k a=1.0$

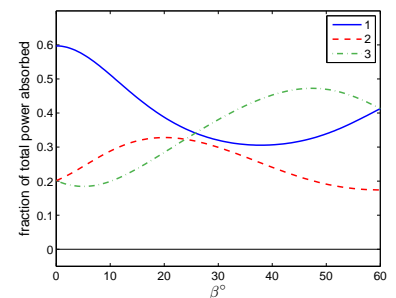

(c) $k a=1.5$

Figure 7: Partition of maximum power amongst wave energy converters for Configuration A, heave-only. 


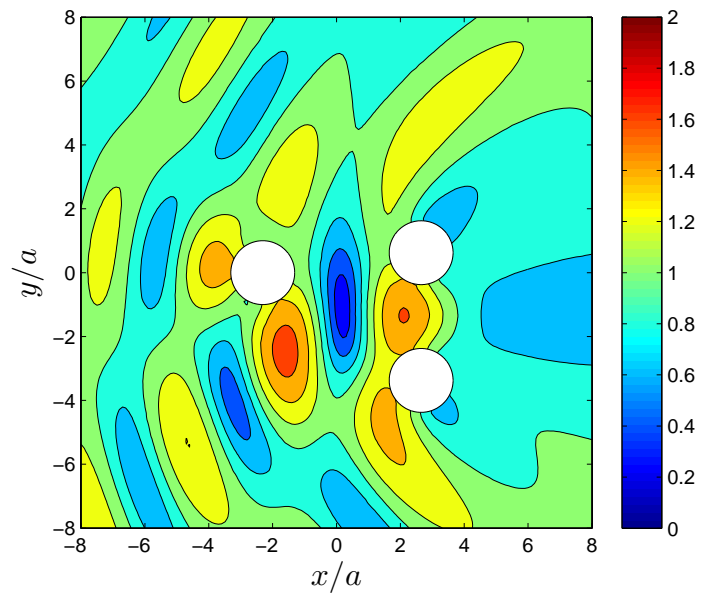

Figure 8: Free surface amplitudes around Configuration $\mathrm{C}$, devices fixed, $k a=1.0 . \quad \beta=0^{\circ}$ (waves incident from left). The colour axis is in multiples of the incident wave amplitude.

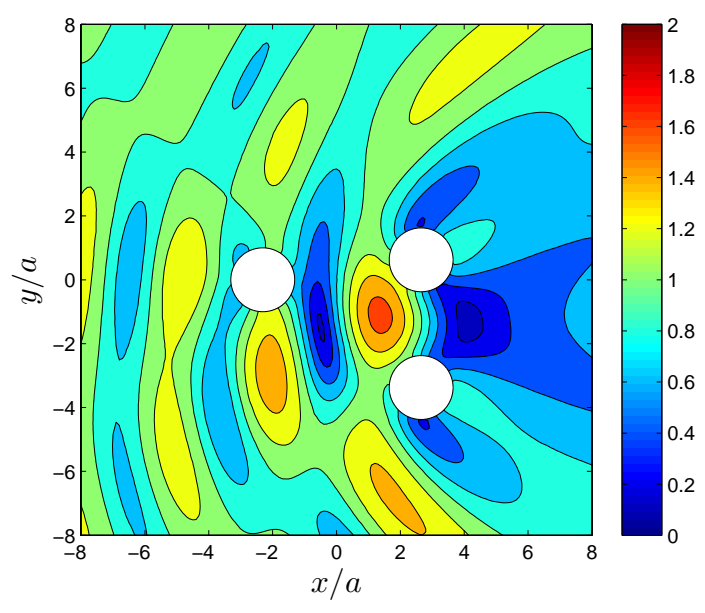

Figure 9: Free surface amplitudes around Configuration $\mathrm{C}$, devices heaving with optimum amplitudes, $k a=1.0 . \quad \beta=0^{\circ}$ (waves incident from left). The colour axis is in multiples of the incident wave amplitude. 


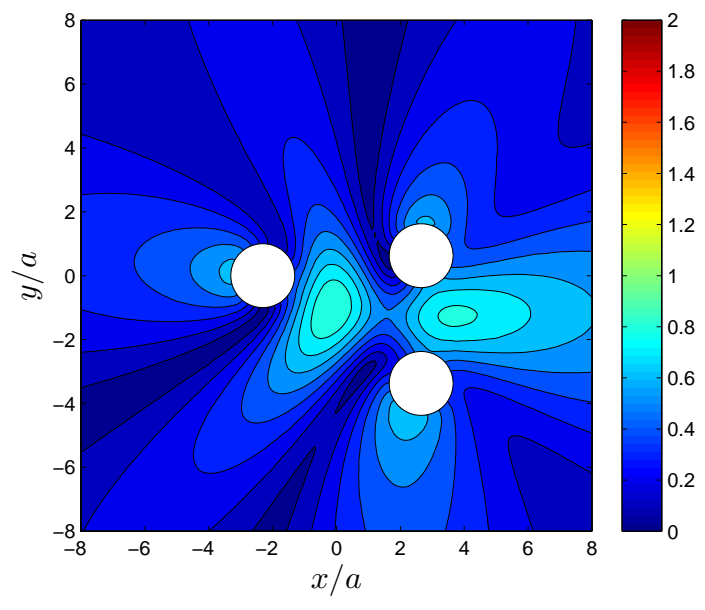

Figure 10: Free surface amplitudes of wave radiated by Configuration $\mathrm{C}$, devices heaving with optimum amplitudes for $\beta=0^{\circ}$, no incident wave, $k a=1.0$. The colour axis is in multiples of the corresponding incident wave amplitude.

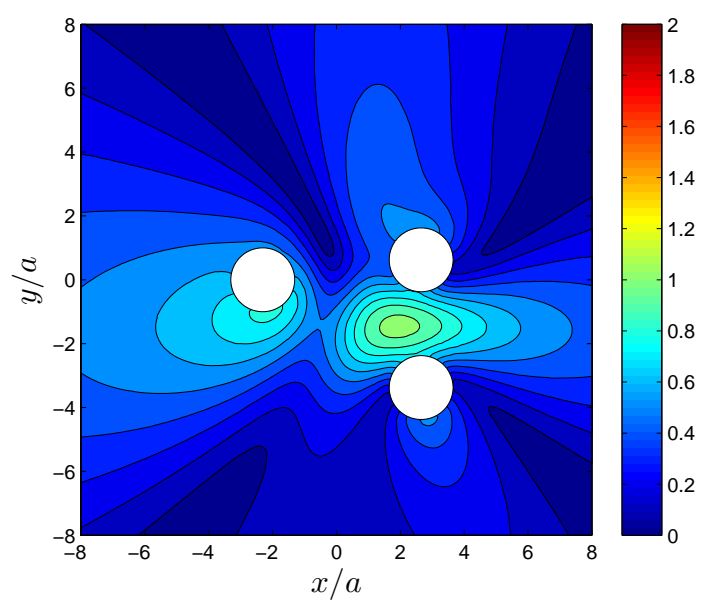

Figure 11: Free surface amplitudes of wave radiated by Configuration C, devices heaving with optimum amplitudes for $\beta=180^{\circ}$, no incident wave, $k a=1.0$. The colour axis is in multiples of the corresponding incident wave amplitude. 


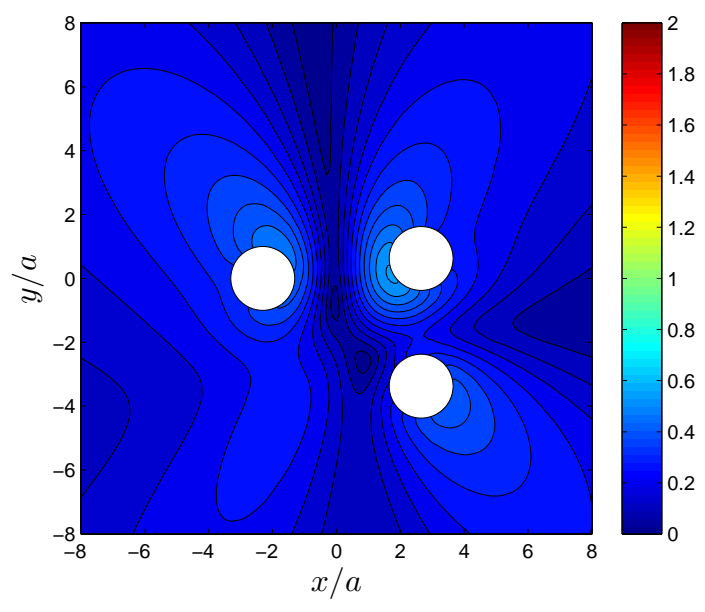

Figure 12: Free surface amplitudes of wave radiated by Configuration $\mathrm{C}$, devices heaving with optimum amplitudes for $\beta=135^{\circ}$, no incident wave, $k a=1.0$. The colour axis is in multiples of the corresponding incident wave amplitude.

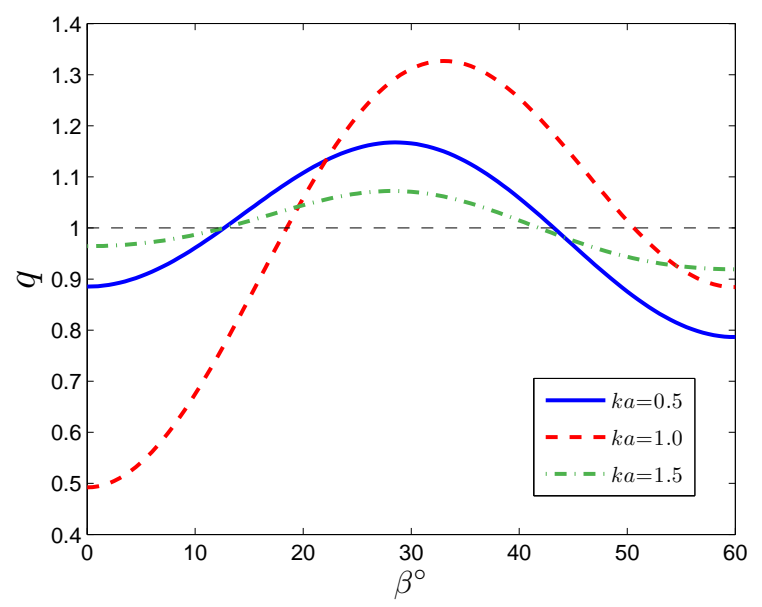

Figure 13: Surge-sway $q$ factor for Configuration A (equilateral triangle). 

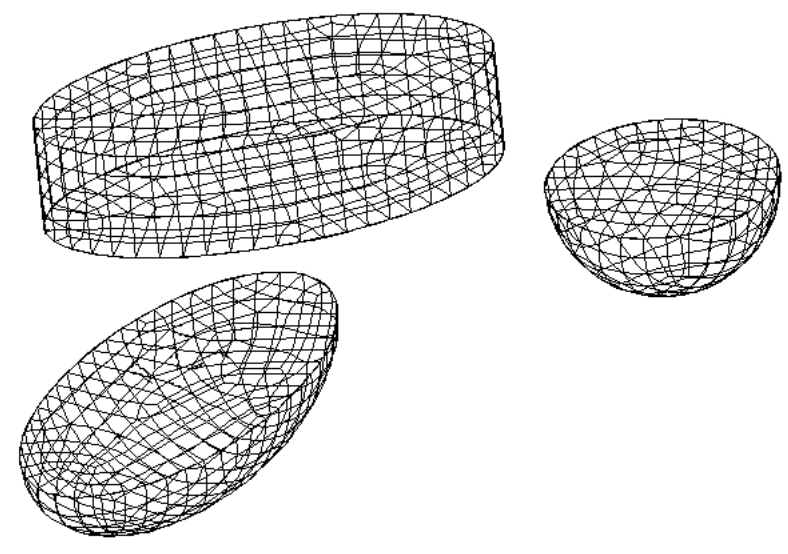

Figure 14: Perspecitve view of meshes for array of arbitrary bodies.

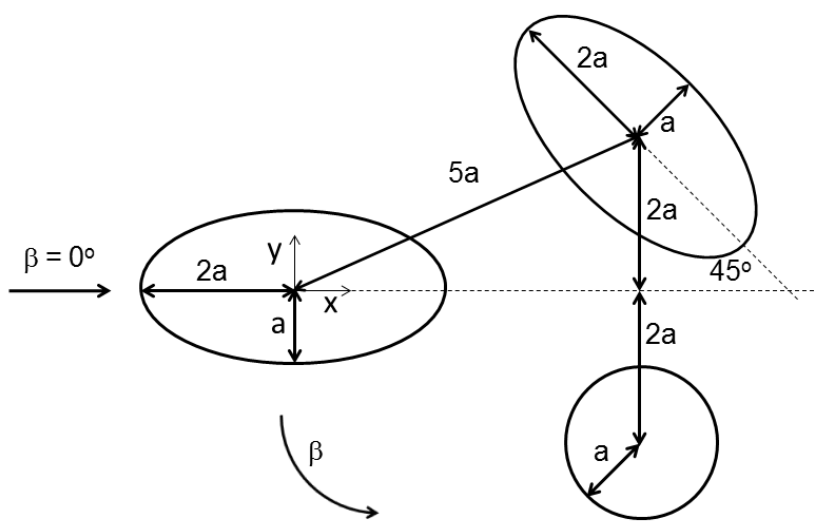

Figure 15: Plan view of array of arbitrary bodies. 


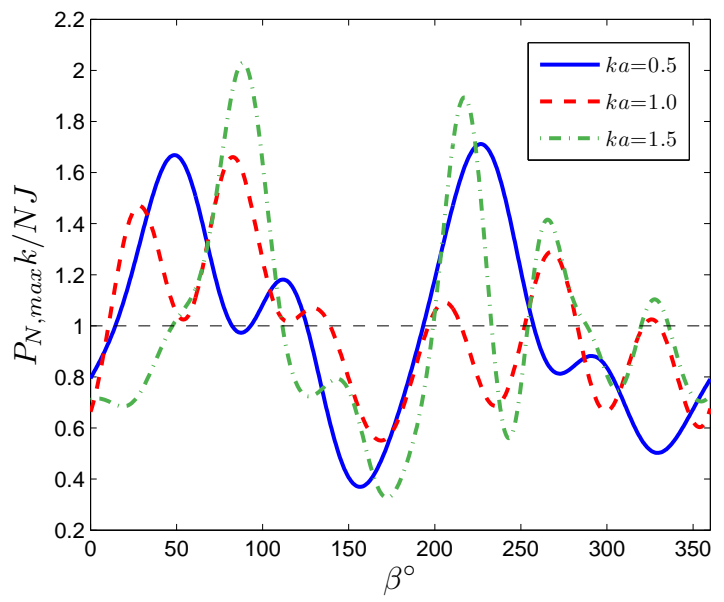

Figure 16: Optimum power absorption by a heterogeneous array. 